\title{
Entwicklung eines Drucksensors mit optischer Auswertung der Membrandeformation
}

\author{
Hartmut Stoltenberg, Herbert Postler
}

In diesem Artikel werden einige Elemente der Entwicklung eines Drucksensors auf der Basis eines neuen, zum Patent angemeldeten, optischen Prinzips dargestellt. Ausgangspunkt für die Entwicklung war die Forderung eines Medizingeräteherstellers nach einer Druckmessung in hochaggressiven Substanzen. Die Messung sollte vorzugsweise an einer leicht zu reinigenden Kunststoffmembran ohne Kavitäten erfolgen. Hinzu kam die Forderung nach Beständigkeit gegen hohe Medientemperaturen.

Durchgefuihrte Recherchen und Untersuchungen gemeinsam mit Partnern ergaben theoretische Ansätze zur Gestaltung der Anordnung und ihrer Umsetzung in drei Hauptbaugruppen. Umfangreiche Entwicklungsarbeiten und Versuche bestätigten die Richtigkeit der Ansätze. Das Funktionsprinzip konnte nachgewiesen werden.

Bevor im Einzelnen auf die Neuentwicklung Bezug genommen wird, soll kurz der Stand der Technik im Bereich Druckmessung/Drucksensorik dargestellt werden.

\section{Prinzipien der Druckmessung}

Die Prinzipien, die zur Messung von Drücken eingesetzt werden, sind ebenso vielfältig wie die Anwendungsbereiche der Druckmessung. Sie reichen von der Füllstandsmessung auf der Grundlage des hydrostatischen Druckes, über die Überwachung von Anlagenzuständen und Fördergeschwindigkeiten in der chemischen Industrie oder auch der Haustechnik bis zu Anwendungen in der Medizintechnik, der Fahrzeugindustrie und der Luftund Raumfahrt.

Die zu messenden Medien unterscheiden sich ebenso wie die relevanten Druckbereiche.

Druckmessung kann in statischen - aber auch in hochdynamischen Betriebsfälle erforderlich sein.

Druck ist definiert als Kraft je Flächeneinheit. In technischen Anwendungen sind Drücke in Flüssigkeiten und Gasen von Interesse.

Jede Druckmessung ist relativ. Messungen erfolgen vielfach bezogen auf den umgebenden Luftdruck. Man spricht in diesem Fall von Gagetypen. Andere Messungen beziehen sich auf Vakuum bzw. in einem Volumen eingeschlossene Referenzdrücke. Ein Sonderfall ist die Differenzdruckmessung. Hierbei ist die Druckdifferenz zwischen zwei Punkten interessant, z. B. um Volumenströme zu bestimmen.

Im Folgenden soll ohne Anspruch auf Vollständigkeit ein Überblick über technische Verfahren der Druckmessung gegeben werden.

\subsection{Flüssigkeitsmanometer}

Das Flüssigkeitsmanometer besteht aus einem flüssigkeitsgefuillten U-Rohr. In dieser Form eignet es sich zum Messen von Druckdifferenzen und von Drücken oberhalb des umgeben Luftdrucks. Wird eine Seite des Rohres verschlossen, sind auch Messungen bezogen auf das Vakuum möglich. Eine Sonderform des Flüssigkeitsmanometers ist das Kolbenmanometer oder auch Druckwaage. Hier wirkt über einen Kolben eine Gewichtskraft auf die Flüssigkeit. Mit einer solchen Anordnung können auch sehr hohe Drücke gemessen werden.

\subsection{Federmanometer}

Federmanometer bestehen aus einem elastischen Element, das sich unter Druckeinwirkung verformt. Im einfachsten Falle handelt es sich dabei um eine Membran, an deren einer Seite ein Referenzdruck anliegt. An der anderen Seite wird der zu messende Druck zugefuihrt. Um den vielfältigen Messaufgaben gerecht zu werden, wurden eine Vielzahl von Lösungen entwickelt. Dazu gehören die verschiedensten Dosen, die je nach Anwendung ein Referenzdruckvolumen enthalten oder mit dem zu messenden Druck gespeist werden. Eine weit verbreitete Form ist das Röhrenfeder-(Bourdonfeder-)Manometer. Dabei handelt es sich um eine einseitig geschlossene, kreisförmig gebogene Röhrenfeder, die sich unter Druck streckt. Diese Streckung wird meist auf entsprechende Zeigerwerke iibertragen.

\subsection{Sonderformen}

Für hohe Drücke ist die Anwendung eines elektrischen Manometers möglich. Ausgenutzt wird, dass ein stromdurchflossener Manganindraht unter hohem Druck seinen Widerstand ändert.

Für Vakuummessungen nutzt man die Änderung des Wärmeleitvermögens oder die Änderung der Ionisationsfähigkeit von Gasen mit abnehmendem Druck.

\section{Drucksensoren, Drucktransmitter}

Ein Sensor erzeugt aus einer physikalischen oder chemischen, allgemein nichtelektrischen Messgröße ein elektrisches Signal. Man unterscheidet den eigentlichen Aufnehmer (Sensor), der die Umsetzung ohne eine aktive Signalaufbereitung vornimmt, den Messumformer (Transmitter), der ausgehend vom Sensorsignal ein definiertes oder genormtes Ausgangssignal bereitstellt und das elektrische Messgerät, das die Messgröße oder einen 
aus ihr abgeleiteten Wert anzeigt und ggf. zur Weiterverarbeitung bereitstellt. In [1] werden elektrische Druckmessgeräte, ihr Aufbau und die zugehörigen Begriffe definiert. Dort werden auch Messprinzipien (u. a. optisch) genannt. Da im vorliegenden Fall der Schwerpunkt auf optische Verfahren gelegt werden soll, werden diese im Folgenden entsprechend hervorgehoben.

\subsection{Nichtoptische Drucksensoren}

Für die Erzeugung eines Messsignals aus der physikalischen Größe Druck sind eine Reihe von Lösungen entwickelt worden. Gemeinsam ist nahezu allen Verfahren, dass die Durchbiegung einer Membran ausgewertet wird. Die Auswertung erfolgt nach piezoresistiven, piezoelektrischen, kapazitiven, induktiven oder DMS-Prinzipien. Diese Prinzipien stellen bestimmte Anforderungen an das Membranmaterial. Membranmaterial ist im allgemeinen Stahl, Keramik oder Silizium. Ist das Messmedium mit diesen Membranen unverträglich, wird mit Vorlagen gearbeitet. Zwischen die eigentliche Messmembran und das Messmedium wird z. B. ein Ölvolumen mit einer zweiten, medienkompatiblen Membran geschaltet.

\subsection{Optische Drucksensoren}

Drucksensoren unter Nutzung optischer Prinzipien spielen gegenwärtig eine untergeordnete Rolle. Sie können wie folgt eingeteilt werden:

\section{- Sensoren, die auf der Verbiegung von Fasern beruhen.} Diese messen die Lichtmenge, die durch die Wand der Faser verloren geht. Sie beinhalten eine Multimodefaser mit Stufenindex. Diese befindet sich auf der Membran. Ein Druck verbiegt die Membran und damit die Faser, was zur Folge hat, dass die Faser an Lichtdurchlässigkeit verliert. Alternativ ist es möglich, polarisiertes Licht durch eine monomodale Faser zu senden. Wird die Faser einer mechanischen Belastung unterzogen, kommt es zu einer Veränderung der internen Reflektionseigenschaften und damit zu einer Drehung der Polarisationsebene. Diese Drehung ist auswertbar.

- Sensoren, die auf der Reflexion von Licht beruhen.

Ein Lichtstrahl wird durch eine Faser senkrecht auf eine Oberfläche (Membran) gesendet. Das reflektierte Licht wird von derselben Faser aufgenommen. Die Intensität des durch die Faser reflektierten Lichtes ändert sich durch die Streuung an der Membranoberfläche in Abhängigkeit von der Entfernung zwischen Faser und Membran, z. B. [11], [12].

- Sensoren, die auf Veränderungen von Transmissionseigenschaften beruhen.

Hier wird durch Druckänderung die Lichtausbreitung mehr oder weniger behindert. Im einfachsten Fall wird durch die Verbiegung der Membran eine Blende in den Lichtstrahl geschoben.

- Sensoren, die interferometrische Prinzipien nutzen. Ein monomodaler Lichtstrahl wird in zwei Strahlen aufgeteilt, einen Messstrahl und einen Referenzstrahl.
Durch die Durchbiegung der Membran wird die Laufzeit des Messstrahls verändert, was sich in einem Phasenunterschied zwischen Messstrahl und Referenzstrahl niederschlägt. Auf diese Weise sind sehr geringe Membranauslenkungen detektierbar. Wenn die Durchbiegung der Membran ein Mehrfaches der Wellenlänge ausmacht, sind jedoch weitere Maßnahmen erforderlich, um ein eindeutiges Messsignal zu erhalten.

Bei allen diesen Sensoren wird entweder die Intensität, die Polarisationsebene oder die Phasenlage eines Lichtstrahls verändert.

\subsection{Prinzip des neuen Drucksensors mit optischer Auswertung der Membrandeformation}

Die Aufgabenstellung erforderte ein Messprinzip, das weitgehend vom verwendeten Membranmaterial unabhängig ist, die Auswertung kleiner Membrandeformationen zulässt, die Messaufgabe möglichst berührungslos realisiert und kostengünstig herstellbare Sensoren ermöglicht.

Die unter 2.1 dargestellten Sensorprinzipien konnten auf Grund der Einschränkungen in der Wahl des Membranmaterials nicht verwendet werden. Die unter 2.2 dargestellten Prinzipien ermöglichen zum großen Teil die berührungslose Messung und eine relativ freie Wahl des Membranmaterials. Sie sind aber auch mit erheblichen Nachteilen behaftet. Diese im Einzelnen fuir jede Variante zu diskutieren, sprengt den Rahmen dieses Artikels. Stellvertretend seien hier zwei Probleme genannt: Eines liegt in den zum Teil erheblichen Aufwenden zur Signalauswertung. Ein zweites liegt in der Notwendigkeit, für verschiedene Anordnungen Alterungserscheinungen der Lichtquelle zu kompensieren.

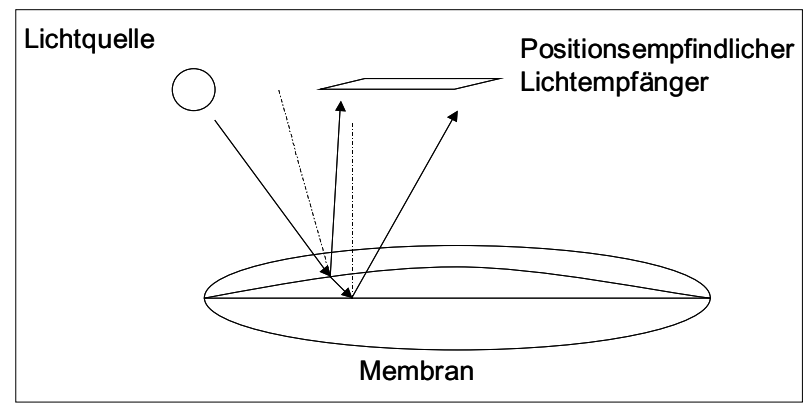

Abb. 1: Prinzip des optischen Drucksensors

Abbildung 1 zeigt die Grundidee des neuen Sensors. Entscheidend ist, dass die Durchwölbung der Membran annähernd „cosinusförmig“ erfolgt. Während in der Mitte der Membran lediglich eine druckabhängige Auslenkung erfolgt, kommt es im Bereich des halben Radius zu einer Überlagerung einer Auslenkung und einer Winkeländerung. Da die Reflexionsachse senkrecht auf der reflektierenden Oberfläche steht, ändert sich der Winkel des reflektierten Strahles um den doppelten Winkel der Membranauslenkung.

Theoretische Untersuchungen [6] zeigen, dass unter der Annahme einer fest eingespannten Membran mit $10 \mathrm{~mm}$ Durchmesser und $40 \mu \mathrm{m}$ Auslenkung eine Strahlaus- 
lenkung von $1.4^{\circ} \mathrm{zu}$ erwarten ist. Unter Ansatz eines Gausstrahles von $1 \mathrm{~mm}$ Durchmesser ergibt sich eine Signalverschiebung in den Emfängerelementen von sechs bis neun Prozent. Für andere Strahlformen und Abmessungen ergeben sich Werte in der gleichen Größenordnung. Bei einem Flat-Top-Profil mit $1 \mathrm{~mm}$ Durchmesser beträgt die Signaländerung mehr als $+/-10 \%$. Es ist ein nahezu linear vom Druck abhängiges Ausgangssignal zu erwarten.

Betrachtet wurden sowohl eine kollimierte LED als auch eine Laserdiode als Strahlungsquelle. Werden andere mechanische Dimensionierungen erforderlich, lassen sich die zu erwartenden Signaländerungen unter Verwendung der Ansätze aus [6] leicht bestimmen.

Ein wesentlicher Schritt zur Realisierung des Sensorprinzips wurde mit einer schrägen Anordnung der optischen Baugruppe im Sensor getan. Dadurch wurde es möglich, die optisch aktiven Elemente in einer Ebene anzuordnen. Das kommt dem Leiterplattenprinzip entgegen und ermöglicht, Lichtsender und Empfänger auf der gleichen Baugruppe zu platzieren.

\section{Realisierung des Sensorprinzips}

Die Realisierung des Sensorprinzips verlangte eine Reihe von Detaillösungen. Da die Zielstellung darin besteht, einen verkaufsfähigen Sensor herzustellen, spielten neben den Fragen von Funktion und Zuverlässigkeit auch Fragen der Herstellbarkeit und Kostenoptimierung eine wichtige Rolle. Es wurde von Anfang an auf minimale Teilezahl und einfache Montage orientiert.

\subsection{Optische Baugruppe}

Die in der Konzeptionsphase entwickelte winklige Anordnung ermöglicht die Anordnung der optisch aktiven Komponenten auf einer Baugruppe. Im Sinne der Miniaturisierung wurde zunächst nach einem Weg gesucht, Lichtsender und Empfänger auf einem Chip unterzubringen. Hier wurde als Partner das CiS Institut für Mikrosensorik gGmbH Erfurt gewonnen. Eine solche Lösung erwies sich als technisch machbar, jedoch ökonomisch im Rahmen des Projektes nicht realisierbar. Als Kompromiss wurde die in Abbildung 2 dargestellte optische Baugruppe entwickelt. Sie besteht aus einem Lichtsender (LED fokussiert durch eine Linse - im linken Teil der abgebildeten

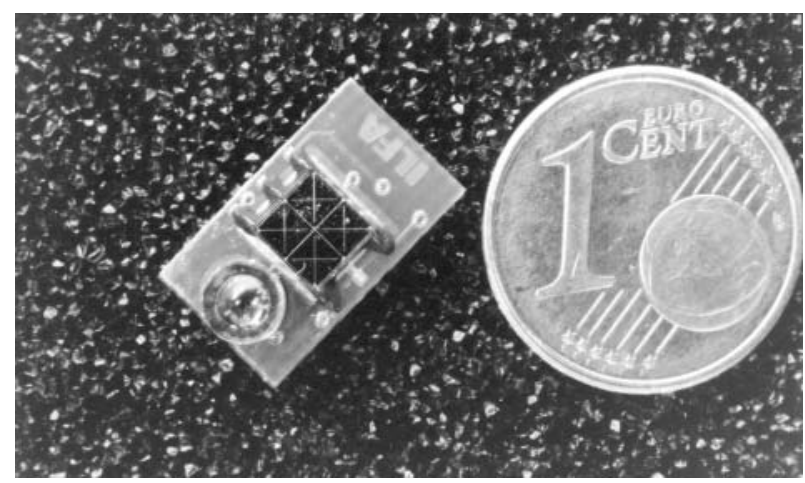

Abb. 2: Optische Baugruppe
Leiterplatte) und Lichtempfänger (Vierquadrantenfotodiode - im rechten Teil der abgebildeten Leiterplatte). Die Kontaktierung erfolgt uiber einen auf der Ruickseite der Leiterplatte angeordneten SMT-Steckverbinder. Durch die gewählte Chip on Board-Technologie konnte eine weitgehende Miniaturisierung erreicht werden.

Parallel zu dieser Entwicklung wurde auch die Laserdiode als Strahlenquelle weiter betrachtet. Laserdioden als Chip standen beim CiS in Erfurt nicht zur Verfügung, so dass eine Lösung mit einer handelsüblichen gehausten Laserdiode und zwei lötbaren Fotodioden als Empfänger entstand. Im Gegensatz zur optischen Baugruppe mit fokussierter LED sind die notwendigen Technologien zur Herstellung der Baugruppe mit Laserdiode bei der Prignitz Mikrosystemtechnik GmbH vorhanden. Ein weiterer Vorteil der Laserdiodenlösung ist der Wegfall der Fokussierung und damit des geklebten Tubusaufbaus. Nachteilig ist das Laserrauschen. Dieser Effekt verursacht ein zusätzliches Störsignal, das die Auswertung behindert.

\subsection{Mechanisches System}

Parallel zur Entwicklung der optischen Baugruppe wurden Entwicklungsarbeiten zum mechanischen System durchgefuihrt. Dabei wurden zwei Grundtypen betrachtet:

- Ein Drucksensor für den frontbündigen Einbau.

- Ein Drucksensor zur Einbindung in ein Schlauchsystem.

Die entsprechenden Lösungsansätze sind in Abbildung 3 und 4 dargestellt.

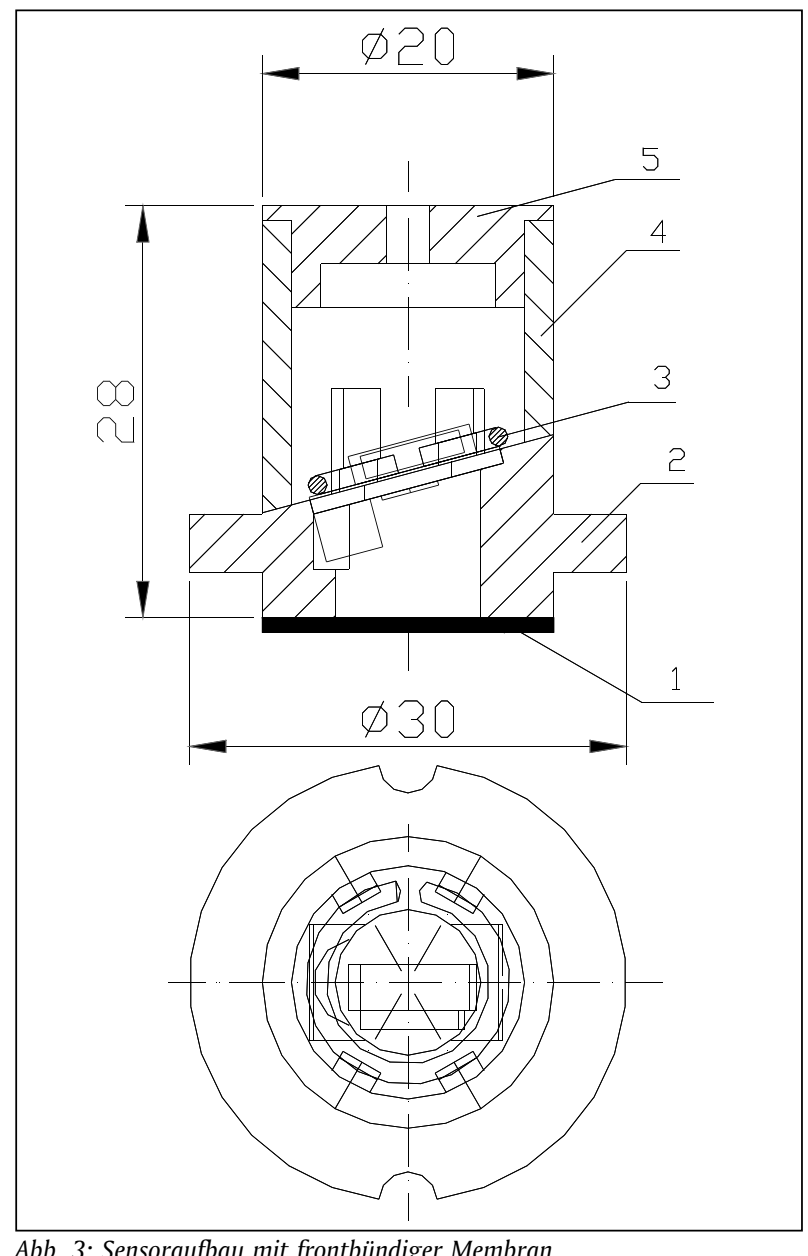

Abb. 3: Sensoraufbau mit frontbündiger Membran 
Das Muster für den frontbündigen Einsatz (Abb. 3) ist mit einem Kragen am Grundkörper (2) ausgestattet, der eine Flanschbefestigung erlaubt. Der Ring (3) fixiert die optische Baugruppe im Grundkörper. Teil (4) gehört zum Grundkörper. Es ist lediglich aus Gründen der Herstellbarkeit für das Muster separiert worden. Wenn der Grundkörper als Spritzgussteil ausgefuihrt ist, werden Teil (2) und (4) als ein Teil gefertigt. Teil (1) ist die Membran. Sie wird ebenfalls mit dem Grundkörper in einem Stück gespritzt werden. Die Auflagekanten im Teil (4) sind für die Aufnahme der Auswerteelektronik vorgesehen. Für diese steht der Raum zwischen dieser Auflagekante und dem Verschlussdeckel (5) zur Verfuigung.

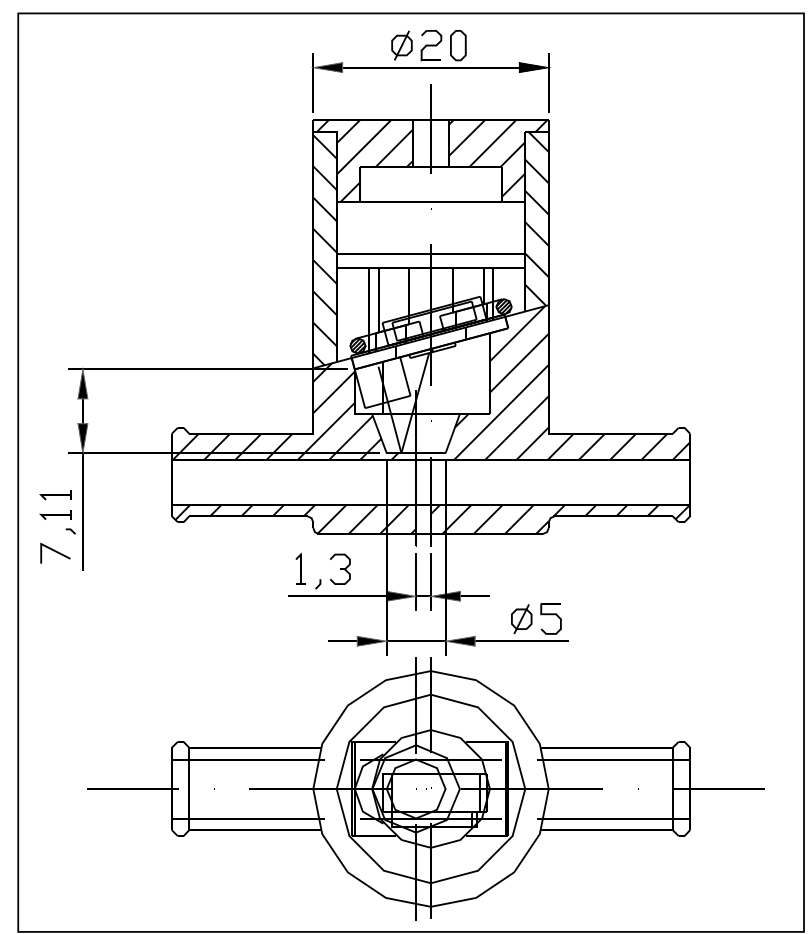

Abb. 4: Sensoraufbau für die Schlauchdruckmessung

Abbildung 4 zeigt die Lösung zur Schlauchdruckmessung. Eine alternative Variante zur Aufnahme der Elektronik, wie sie ach im Muster realisiert worden ist, zeigt Abbildung 5. Hier wird der Winkel der optischen Bau-

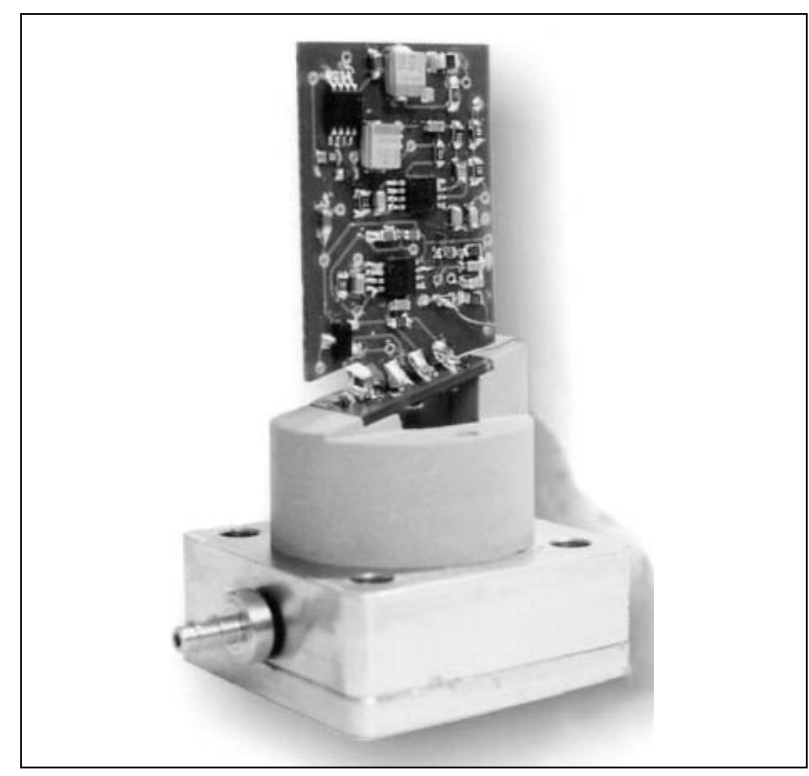

Abb. 5: Musteraufbau des optischen Drucksensors (ohne Gehäuse) gruppe in der Leiterplatte aufgenommen, was eine unmittelbare Lötverbindung zwischen optischer Baugruppe und Leiterplatte erlaubt. Auf diese Weise sind zusätzliche Verbindungselemente in Form von Kabeln, flexiblen Leiterplatten oder ähnlichem entbehrlich.

Die Fertigung der Muster erfolgte in zwei Varianten. Neben der ursprünglichen Variante in Kunststoff entstand ein Muster mit Edelstahlgehäuse und Edelstahlmembran. Wie unter 2.3 ausgefuihrt, kann die Wahl des Membranmaterials weitgehend frei erfolgen. Es gibt jedoch Einschränkungen. Die erste Einschränkung ist, dass es optisch reflektierende Eigenschaften haben muss. Dabei ist keine ideale Reflexion erforderlich, es muss aber ein deutlicher Strahlschwerpunkt auszuwerten sein. Unter Beachtung der Bearbeitungsrichtung ist diese Forderung mit Edelstahl leicht zu erfuillen. Soweit Kunststoffe diese Forderung nicht erfüllen, ist es möglich, mittels entsprechender Beschichtung Abhilfe zu schaffen. Die zweite Einschränkung ist die Forderung nach Membraneigenschaften. Membraneigenschaften bedeutet an dieser Stelle, dass die Membran unter Druckbelastung definiert verformt wird, nach Entlastung aber wieder in den Ausgangszustand zurückkehrt. Diese Eigenschaft wird von Edelstahl erfuillt. Kunststoffe zeigen jedoch eine Reihe von Eigenschaften, u. a. Kriechen, die zu Hysteresen und anderen Effekten fuihren. Diese Problematik wird noch Gegenstand ausführlicher Untersuchungen sein müssen, um hier zu befriedigenden Lösungen zu kommen. Erste Kontakte mit Partnern sind hierzu aufgenommen.

\subsection{Elektronische Signalverarbeitung}

Die optische Baugruppe ist mit Strom zu versorgen. Ihre Ausgangssignale müssen aufbereitet, korrigiert und mit der entsprechenden Schnittstellennorm für den Sensoranwender bereitgestellt werden. Idealerweise sollte die Elektronik keine Leistung aufnehmen, keinen Raum einnehmen, temperaturunabhängig arbeiten, alle im System auftretenden Fehler kompensieren, einfach und mit Standardbauelementen montierbar sein und nichts kosten. Der fertige Transmitter soll effektiv, unter Einsatz von Computerlösungen kalibrierbar und ggf. auch nachkalibrierbar sein.

Für die Realisierung des optischen Prinzips ist eine gewisse Leistung unverzichtbar. Sowohl die Lösung mit LED als auch die Lösung mit Laserdiode benötigt zur Erzeugung des notwendigen Lichtes Strom. Es muss sichergestellt werden, dass die Fotodioden hinreichend beleuchtet werden, um Fotoströme zu erzeugen, die weit genug uiber den Dunkelströmen der Fotodioden liegen, um rauscharme und weitgehend von thermischen Effekten unbeeinflusste Signale zu erhalten. Die gegenwärtig zur Verfügung stehenden Bauelemente gestatten nicht, einen optischen 4-20 mA Sensor als Zweidrahtlösung herzustellen.

Neben der Frage der elektronischen Bauelemente stellt sich hier natuirlich auch nochmals die Frage nach der Optimierung des optischen Systems. Um eine gute Abdeckung der Empfängerelemente mit dem Lichtkegel zu 
erreichen, muss u. a. die Oberfläche der Empfängerelemente optimiert werden. Der Lichtkegel sollte einen bestimmten Gradienten aufweisen. Das fuihrt zu besser auswertbaren Ergebnissen als ein scharfer Lichtpunkt.

Die Ausgangssignale der Photodioden liegen im $\mu \mathrm{A}-$ bis nA-Bereich. Um sie nutzbar zu machen, ist eine effektive Signalaufbereitung notwendig. Nach einer ersten Verstärkung werden sie einem Standardsensorschaltkreis zugefuihrt. Dieser Schaltkreis hat eine Reihe von Aufgaben. Die Wichtigste ist die der Fehlerkompensation. Auf Grund von Toleranzen im Herstellungsprozess differieren die Ausgangssignale von Sensor zu Sensor. Im Zuge eines Kalibrierprozesses werden Korrekturfaktoren für den Nullpunktoffset, Verstärkung, Temperaturoffset und eine temperaturabhängige Verstärkungskorrektur ermittelt und in den Standardsensorschaltkreis eingeschrieben. Zur Speicherung dieser Daten verfuigt der Schaltkreis über einen EEPROM-Bereich, in dem diese Daten ausfallsicher gespeichert werden. Abhängig vom Typ sind weitere Korrekturen (z. B. abschnittsweise Linearitätskompensation) möglich. Das Blockdiagramm des in den Mustern eingesetzten Schaltkreises ist in Abbildung 6 dargestellt.

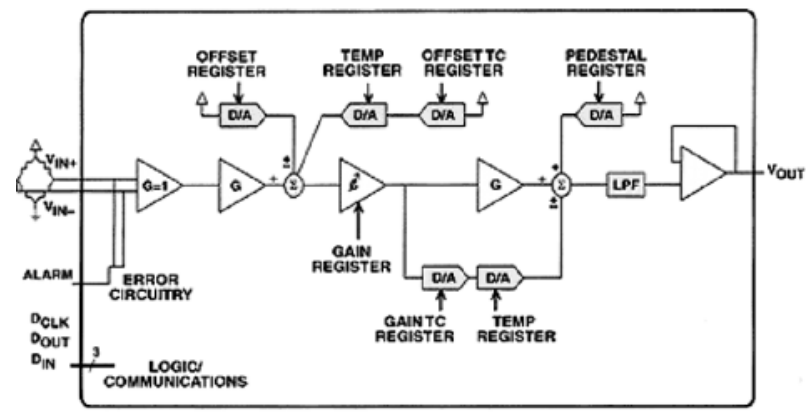

Abb. 6: Blockschaltbild des Standardsensorschaltkreises

Der Kalibrierprozess wird zukünftig automatisiert ablaufen. Ein Rechner steuert die Bereitstellung von Referenzdrücken unter den entsprechenden Temperaturbedingungen, liest die Sensorsignale aus, ermittelt die Korrekturwerte und speichert diese im Schaltkreis ab. Das Ausgangssignal des Standardsensorschaltkreises wird mit einer Endstufe dem Ausgangssignalwunsch des Kunden angepasst. Für den Fall eines digitalen Ausgangsignals ist die Verwendung eines Mikrocontrollers vorgesehen.

Die Schaltungsentwicklung in diesem Bereich ist noch nicht abgeschlossen.

\section{Erreichter Stand}

Der Prototyp des Sensors zur Messung von Drücken im Bereich von 0 bis 1 bar mit einem Ausgangssignal von 0 bis $10 \mathrm{~V}$ konnte auf der Hannovermesse Industrie 2003 der Öffentlichkeit vorgestellt werden. Die Resonanz zeigte, dass die Konzentration auf eine frontbündige Lösung richtig war. Sie traf das Interesse der Besucher. Dabei konnten eine Reihe weiterer Anregungen zu ihrer Gestaltung aufgenommen werden. Großes Interesse galt auch der Entkopplung von Membran und Elektronik und dem damit verbundenen erweiterten Temperaturbereich. Konkrete Untersuchungen zu den erreichbaren Temperaturgrenzen stehen noch aus. Großes Interesse herrscht auch an der Druckmessung im mbar-Bereich. In diesem Zusammenhang steht die Frage nach einer geeigneten Membrankonstruktion mit hoher Überlastfestigkeit. Dazu ist die Untersuchung geeigneter Kunststoffe fortzusetzen.

Neben der vorgestellten Variante mit einem Ausgangssignal von 0 bis $10 \mathrm{~V}$ sind die elektronischen Baugruppen fuir Varianten mit 4 bis $20 \mathrm{~mA}$, RS485, PWM und CAN-Bus vorbereitet worden. Sie sind jetzt in Betrieb zu nehmen und den Kunden vorzustellen.

Mit dem Prototyp des Sensors ist ein wichtiger Teil des Weges zurüickgelegt worden. Bis zu einer Serienproduktion des Sensors sind aber noch eine große Zahl von Aufgaben zu erfuillen und eine Reihe von Voraussetzungen zu schaffen.

\section{Literatur}

[1] DIN 16086, Elektrische Druckmessgeräte, 1992

[2] Pepperl, R.: Optische Abstandsmessung, Haus der Technik Fachbuchreihe, Vulkan-Verlag (1993)

[3] Krieg, B.: Automatisieren mit Optoelektronik, Vogel Verlag (1992)

[4] Schiessle, E.: Sensortechnik und Messwertaufnahme, Vogel Verlag (1992)

[5] Sohler, W.; Volk, R.: Integrierte Optik - Potential für mittelständische Hersteller und Anwender von Mikrosystemen, VDI/VDE Technologiezentrum Informationstechnik GmbH

[6] Steiger, B.: Abschlußbericht Voruntersuchungen Optischer Sensor, unveröffentlicht (2002)

[7] Stoltenberg, H.: Zwischenbericht zum PuV-Projekt „Optische Vorrichtung zum Messen des Druckes“ unveröffentlicht (2002)

[8] Grudzien, Jr.: Optical Pressure Transducer, U.S.Patent 5,127,296 07.07 (1992)

[9] Theobald, S.A..; Torcy, FR.; Differential-Drucksensor, Gebrauchsmuster (1995)

[10] Barkhoudarian, Sarkis u.a.; Optical Presssure Sensor, U.S.Patent 4,620,093 (1986)

[11] Hoffman, R. u.a.: Optical Pressure Sensor, WO 86/07445 (1986)

[12] Hsu, Tsung-Yuan,: Fiber optic high temperature pressure sensor, EP 1026493 A2 (1994)

\section{Autoren}

\section{Dr.-Ing. Hartmut Stoltenberg}

Technische Fachhochschule Wildau

Technologietransfer und Innovationsberatung

Telefon +493877984124

E-Mail: hstolten@tf.tfh-wildau.de

\section{Dipl.-Ing. Herbert Postler}

Prignitz Mikrosystemtechnik $\mathrm{GmbH}$

Laborstraße 1, 19322 Wittenberge

Telefon +493877984124

E-Mail: info@prignitz-mst.de

Das diesem Bericht zugrunde liegende Vorhaben wurde mit Mitteln des Landes Brandenburg gefördert. Die Verantwortung für den Inhalt der Veröffentlichung liegt beim Autor. 